\title{
A filtration on the ring of Laurent polynomials and representations of the general linear Lie algebra
}

\author{
C. Choi, S. Kim, and H. Seo \\ Communicated by A. P. Petravchuk
}

\begin{abstract}
A BStRACT. We first present a filtration on the ring $L_{n}$ of Laurent polynomials such that the direct sum decomposition of its associated graded ring $g r L_{n}$ agrees with the direct sum decomposition of $g r L_{n}$, as a module over the complex general linear Lie algebra $\mathfrak{g l}(n)$, into its simple submodules. Next, generalizing the simple modules occurring in the associated graded $\operatorname{ring} g r L_{n}$, we give some explicit constructions of weight multiplicity-free irreducible representations of $\mathfrak{g l}(n)$.
\end{abstract}

\section{Introduction}

In this section, we give a brief summary of our results.

\subsection{The ring of polynomials}

The ring $P_{n}=\mathbb{C}\left[x_{1}, \ldots, x_{n}\right]$ of polynomials in $n$ indeterminates over the complex numbers $\mathbb{C}$ is a $\mathbb{Z}$-graded algebra

$$
P_{n}=\bigoplus_{m \in \mathbb{Z}} P_{n}^{(m)}
$$

2020 MSC: 16S34, 16W70, 17B10, 17B45.

Key words and phrases: Laurent polynomial, filtration, general linear Lie algebra, weight module. 
where $P_{n}^{(m)}$ is the space of homogeneous polynomials of degree $m$. As a vector space, $P_{n}$ becomes a module over the complex general linear Lie algebra $\mathfrak{g l}(n)=\mathfrak{g l}_{n}(\mathbb{C})$ under the action

$$
A \cdot f=\sum_{i j} a_{i j} x_{i} \frac{\partial f}{\partial x_{j}} \quad \text { for } A=\left(a_{i j}\right) \in \mathfrak{g l}(n) \text { and } f \in P_{n} .
$$

Then, the direct sum decomposition (1.1) of $P_{n}$ as a graded ring agrees with the decomposition of $P_{n}$ as a $\mathfrak{g l}(n)$-module into its simple submodules $P_{n}^{(m)}$. They are the finite dimensional representations of $\mathfrak{g l}(n)$ labeled by Young diagrams with single rows.

\subsection{The ring of Laurent polynomials}

The first goal of this paper is to obtain an analogous result of the above observation for the ring of Laurent polynomials

$$
L_{n}=\mathbb{C}\left[x_{1}^{ \pm 1}, x_{2}^{ \pm 1}, \ldots, x_{n}^{ \pm 1}\right] .
$$

It turns out that a filtration and its associated graded structure give us an answer. Note that (1.1) can be seen as the graded ring associated with the $\mathbb{Z}$-filtration of $P_{n}$ given by degree.

We will define a filtration on $L_{n}$ by a partially ordered monoid constructed from integers and subsets of $\{1,2, \ldots, n\}$

$$
L_{n}=\bigcup_{(m, J) \in \mathbb{Z} \times \mathscr{P}_{n}} L_{n}^{\leqslant(m, J)}
$$

and show that the direct sum decomposition of its associated graded ring

$$
g r L_{n}=\bigoplus_{(m, J) \in \mathbb{Z} \times \mathscr{P}_{n}} L_{n}^{\leqslant(m, J)} / L_{n}^{<(m, J)}
$$

provides the decomposition of $g r L_{n}$, as a $\mathfrak{g l}(n)$-module, into its simple submodules.

Extending the space with the action (1.2) of $\mathfrak{g l}(n)$ from $P_{n}$ to $L_{n}$, we identify Laurent monomials $\mathbf{x}^{\mathbf{k}}=x_{1}^{k_{1}} x_{2}^{k_{2}} \cdots x_{n}^{k_{n}}$ with integral points $\mathbf{k}=\left(k_{1}, k_{2} \ldots, k_{n}\right)$ in $\mathbb{R}^{n}$. Note that they are weight vectors with respect to the Cartan subalgebra of $\mathfrak{g l}(n)$ consisting of diagonal matrices. Since this action preserves the degree of monomials, we can focus on integral points on the hyperplane $k_{1}+\cdots+k_{n}=m$ for each $m \in \mathbb{Z}$.

One of main difficulties in studying the $\mathfrak{g l}(n)$-module structure of $L_{n}$ is that the symmetric behavior of raising and lowering operators we had 


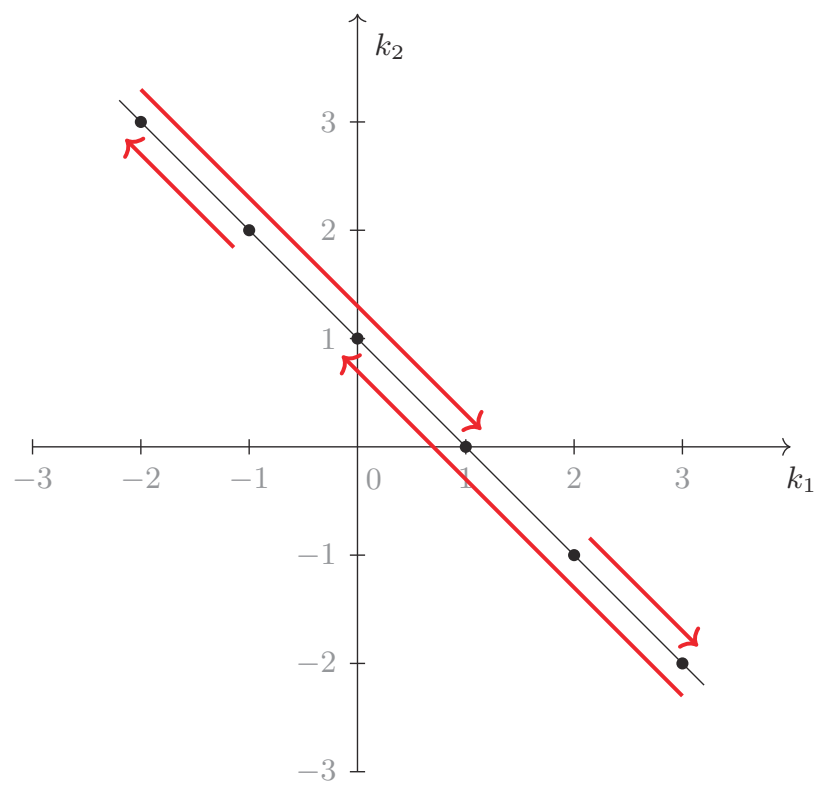

FIGURE 1 . The action of $\mathfrak{g l}(2)$ on $x_{1}^{k_{1}} x_{2}^{k_{2}}$ with $k_{1}+k_{2}=1$.

when working with $P_{n}$ is not trivial anymore. For example, when $n=2$ as in Figure 1,

$$
\begin{aligned}
& {\left[\begin{array}{ll}
0 & 0 \\
1 & 0
\end{array}\right] \cdot x_{1}^{k_{1}} x_{2}^{m-k_{1}}=k_{1} x_{1}^{k_{1}-1} x_{2}^{m+1-k_{1}},} \\
& {\left[\begin{array}{ll}
0 & 1 \\
0 & 0
\end{array}\right] \cdot x_{1}^{m-k_{2}} x_{2}^{k_{2}}=k_{2} x_{1}^{m+1-k_{2}} x_{2}^{k_{2}-1} .}
\end{aligned}
$$

The cases $k_{1}=0$ and $k_{2}=0$ divide the line $k_{1}+k_{2}=m$ into three parts. The monomials with $k_{1} \geqslant 0$ and $k_{2} \geqslant 0$ can be obtained by applying some elements of $\mathfrak{g l}(2)$ to monomials with $k_{1} k_{2}<0$. However, monomials with $k_{1} k_{2}<0$ cannot be obtained from the ones with $k_{1} \geqslant 0$ and $k_{2} \geqslant 0$.

More generally, the planes $k_{j}=0$ divide the hyperplane $k_{1}+k_{2}+\cdots+$ $k_{n}=m$ into regions labeled by the signs of the coordinates $k_{i}$. Then, for each $i$, we can obtain weight vectors $\mathbf{x}^{\mathbf{k}}$ with $k_{i} \geqslant 0$ starting from the ones with $k_{i}<0$ by successively applying some elements of $\mathfrak{g l}(n)$, but the opposite way is not possible. See Figure 2.

Therefore, our indecomposable submodules in $L_{n}$ and simple modules obtained from their quotients are labeled by degree $m$ of $\mathbf{x}^{\mathbf{k}}$ and subsets $J$ of $\{1,2, \ldots, n\}$ indicating the position of possible negative components 


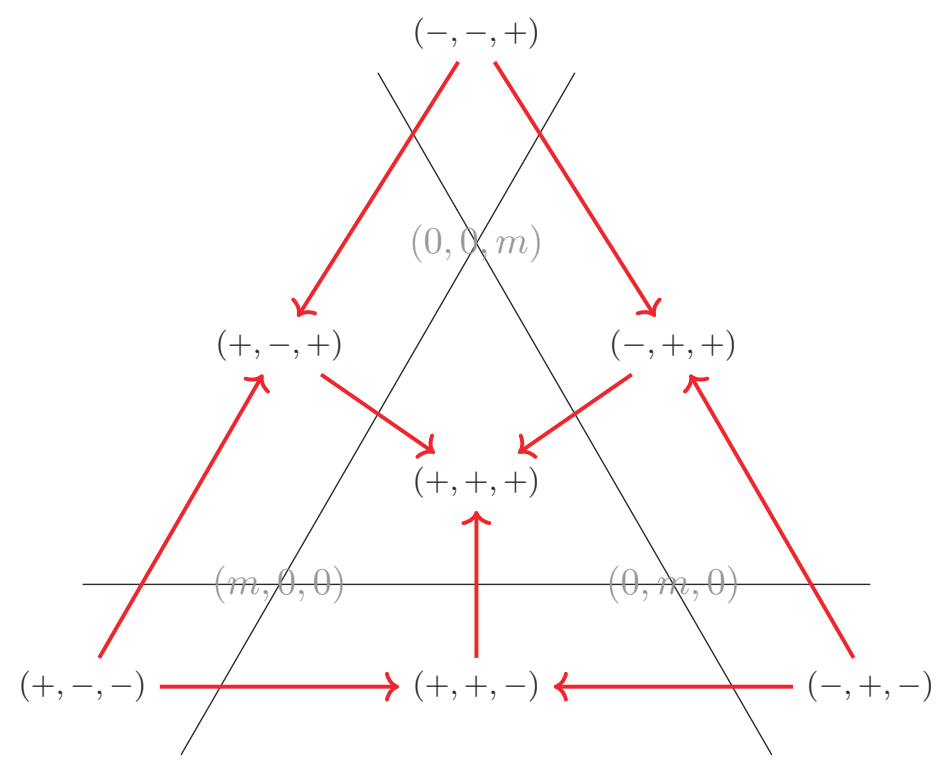

FIgURE 2. The action of $\mathfrak{g l}(3)$ on $x_{1}^{k_{1}} x_{2}^{k_{2}} x_{3}^{k_{3}}$ with $k_{1}+k_{2}+k_{3}=m(m>0)$.

in $\mathbf{k}$. Their structures depend heavily on $m$ and the cardinality of $J$. We will give a clear case-by-case analysis of them.

\subsection{Representations of the general linear Lie algebra}

Our next goal is to provide explicit constructions of weight multiplicityfree irreducible representations of $\mathfrak{g l}(n)$ obtained by twisting the action (1.2). For a general theory on weight multiplicity-free representations of simple Lie algebras, see [1] and references therein.

Motivated by works on weight modules of the Lie algebra of diffeomorphisms of the $n$-dimensional torus (see, for example, [3, 4, 6]), for each $\boldsymbol{\alpha}=\left(\alpha_{1}, \ldots, \alpha_{n}\right) \in \mathbb{C}^{n}$, we will define a representation $L_{n}^{\boldsymbol{\alpha}}$ of $\mathfrak{g l}(n)$ on the vector space $L_{n}$ (see Definition 3.2). Then, we investigate two families of its submodules, $L_{n}^{\boldsymbol{\alpha}}(m, j)$ and $V_{n}^{\boldsymbol{\alpha}}(m, J)$, parameterized by integers $m, j$, and subsets $J$ of $\left\{i: \alpha_{i}=0\right\}$. We can obtain explicit simple $\mathfrak{g l}(n)$-modules from the decomposition of the quotient modules

$$
L_{n}^{\boldsymbol{\alpha}}(m, j) / L_{n}^{\boldsymbol{\alpha}}(m, j-1)=\bigoplus_{J:|J|=j} W_{n}^{\boldsymbol{\alpha}}(m, J)
$$

where $W_{n}^{\boldsymbol{\alpha}}(m, J)$ are simple modules defined by

$$
W_{n}^{\boldsymbol{\alpha}}(m, J)=\left(V_{n}^{\boldsymbol{\alpha}}(m, J)+L_{n}^{\boldsymbol{\alpha}}(m, j-1)\right) / L_{n}^{\boldsymbol{\alpha}}(m, j-1) .
$$


Among these simple modules, there are highest weight modules with highest weights of the form $\psi^{\lambda} \in \mathfrak{h}^{*}$ where

$$
\lambda=(-1, \ldots,-1, z, 0, \ldots, 0) \in \mathbb{C}^{n}
$$

including the finite dimensional ones having integral dominant weights with $\lambda=(k, 0, \ldots, 0)$ and $(-1, \ldots,-1, \ell)$ for $k \geqslant 0$ and $\ell \leqslant-1$.

\section{A filtration on $L_{n}$ and simple modules in $g r L_{n}$}

In this section, we impose a filtration on the ring

$$
L_{n}=\mathbb{C}\left[x_{1}^{ \pm 1}, \ldots, x_{n}^{ \pm 1}\right]
$$

of Laurent polynomials in $n$ indeterminates over the complex numbers $\mathbb{C}$, and then show that the graded structure of its associated graded ring is compatible with the module structure of $L_{n}$ over the complex general linear Lie algebra $\mathfrak{g l}(n)=\mathfrak{g l}_{n}(\mathbb{C})$.

Recall that $\mathfrak{g l}(n)$ is the Lie algebra of $n \times n$ complex matrices with the usual matrix addition and the Lie bracket given by the commutator of two matrices. We will write $\mathcal{U}_{n}=\mathcal{U}(\mathfrak{g l}(n))$ for the universal enveloping algebra of $\mathfrak{g l}(n)$.

\subsection{Submodules of $L_{n}$}

The complex vector space $L_{n}$ is spanned by monomials

$$
\mathbf{x}^{\mathbf{k}}=x_{1}^{k_{1}} x_{2}^{k_{2}} \cdots x_{n}^{k_{n}}
$$

for $\mathbf{k}=\left(k_{1}, k_{2}, \ldots, k_{n}\right) \in \mathbb{Z}^{n}$. We define some subspaces of $L_{n}$.

Definition 2.1. Let $m$ be an integer, $j$ be an integer with $0 \leqslant j \leqslant n$, and $J$ be a subset of $\{1,2, \ldots, n\}$.

1) Let $V_{n}(m, J)$ be the subspace of $L_{n}$ spanned by all the monomials $x_{1}^{k_{1}} x_{2}^{k_{2}} \cdots x_{n}^{k_{n}}$ such that

$$
\sum_{i=1}^{n} k_{i}=m \quad \text { and } \quad\left\{i: k_{i}<0\right\} \subseteq J .
$$

2) Let $L_{n}(m, j)$ be the sum of the subspaces $V_{n}(m, J)$ of $L_{n}$

$$
L_{n}(m, j)=\sum_{J:|J|=j} V_{n}(m, J)
$$

over all subsets $J$ of $\{1,2, \ldots, n\}$ having $j$ elements. 
It follows directly from the definition that

$$
V_{n}\left(m, J_{1}\right) \subseteq V_{n}\left(m, J_{2}\right) \quad \text { for all } J_{1} \subseteq J_{2} .
$$

Then, we have

$$
L_{n}(m, j-1) \subseteq L_{n}(m, j)
$$

and their quotient can be expressed as

$$
\begin{aligned}
L_{n}(m, j) / L_{n}(m, j-1) & =\left(\sum_{J:|J|=j} V_{n}(m, J)\right) / L_{n}(m, j-1) \\
& =\sum_{J:|J|=j}\left(V_{n}(m, J)+L_{n}(m, j-1)\right) / L_{n}(m, j-1) .
\end{aligned}
$$

Definition 2.2. For an integer $m$ and a subset $J$ of $\{1,2, \ldots, n\}$ having $j$ elements, we let $W_{n}(m, J)$ denote the subspace

$$
W_{n}(m, J)=\left(V_{n}(m, J)+L_{n}(m, j-1)\right) / L_{n}(m, j-1)
$$

of the quotient space $L_{n}(m, j) / L_{n}(m, j-1)$.

The spaces $V_{n}(m, J), L_{n}(m, j)$, and $W_{n}(m, J)$ are special cases of the ones defined in Definition 3.5 and Definition 6.1 with $\boldsymbol{\alpha}=\mathbf{0}$ and $I_{\boldsymbol{\alpha}}=\{1,2, \ldots, n\}$, and they are modules over $\mathcal{U}_{n}$ with respect to

$$
A \cdot f=\sum_{i j} a_{i j} x_{i} \frac{\partial f}{\partial x_{j}}
$$

for $A=\left(a_{i j}\right) \in \mathfrak{g l}(n)$ and $f \in L_{n}$. See Theorems 4.4, 4.5, 5.4, and 5.5. Moreover, $W_{n}(m, J)$ are simple modules. See Theorem 6.2.

Lemma 2.3. For $m \in \mathbb{Z}$ and a subset $J$ of $\{1,2, \ldots, n\}$ having $j$ elements, as a $\mathcal{U}_{n}$-module,

$$
W_{n}(m, J) \cong V_{n}(m, J) / \sum_{J^{\prime}} V_{n}\left(m, J^{\prime}\right)
$$

where the summation is over all subsets $J^{\prime}$ of $J$ having $j-1$ elements Proof. Note that

$$
\begin{aligned}
W_{n}(m, J) & =\left(V_{n}(m, J)+L_{n}(m, j-1)\right) / L_{n}(m, j-1) \\
& \cong V_{n}(m, J) /\left(V_{n}(m, J) \cap L_{n}(m, j-1)\right) .
\end{aligned}
$$

Then, the statement follows from the following observation

$$
V_{n}(m, J) \cap L_{n}(m, j-1)=\sum_{J^{\prime}} V_{n}\left(m, J^{\prime}\right)
$$

where the sum is over all subsets $J^{\prime}$ of $J$ having $j-1$ elements. 


\subsection{Filtration by a partially ordered monoid}

Let $\mathscr{P}_{n}$ be the set of all subsets of $\{1,2, \ldots, n\}$. On the set $\mathbb{Z} \times \mathscr{P}_{n}$, we define the partial order $\left(m_{1}, J_{1}\right) \leqslant\left(m_{2}, J_{2}\right)$ if $m_{1} \leqslant m_{2}$ and $J_{1} \subseteq J_{2}$, and the multiplication

$$
\left(m_{1}, J_{1}\right) *\left(m_{2}, J_{2}\right)=\left(m_{1}+m_{2}, J_{1} \cup J_{2}\right) .
$$

With $\leqslant$ and $*, \mathbb{Z} \times \mathscr{P}_{n}$ becomes a partially ordered monoid with the identity $(0, \varnothing)$, and using this monoid we want to impose a filtration on $L_{n}$. For basic properties of a filtration of a ring given by a partially ordered monoid, we refer to $[2, \S I .12]$.

Definition 2.4. For each $(m, J) \in \mathbb{Z} \times \mathscr{P}_{n}$, we define

$$
L_{n}^{\leqslant(m, J)}=\sum_{\left(m_{1}, J_{1}\right)} V_{n}\left(m_{1}, J_{1}\right) \quad \text { and } \quad L_{n}^{<(m, J)}=\sum_{\left(m_{2}, J_{2}\right)} V_{n}\left(m_{2}, J_{2}\right)
$$

where the first summation is over all $\left(m_{1}, J_{1}\right)$ such that

$$
\left(m_{1}, J_{1}\right) \leqslant(m, J),
$$

and the second summation is over all $\left(m_{2}, J_{2}\right)$ such that

$$
\left(m_{2}, J_{2}\right) \leqslant(m, J) \text { but }\left(m_{2}, J_{2}\right) \neq(m, J) .
$$

Proposition 2.5. The family $\left\{L_{n}^{\leqslant(m, J)}:(m, J) \in \mathbb{Z} \times \mathscr{P}_{n}\right\}$ of subspace of $L_{n}$ defines a filtration on $L_{n}$ by the partially ordered monoid $\mathbb{Z} \times \mathscr{P}_{n}$.

Proof. We need to check the following conditions (see [2, §I.12]).

1) $1 \in L_{n}^{\leqslant(0, \varnothing)}$,

2) for all $\left(m_{1}, J_{1}\right) \leqslant\left(m_{2}, J_{2}\right)$,

$$
L_{n}^{\leqslant\left(m_{1}, J_{1}\right)} \subseteq L_{n}^{\leqslant\left(m_{2}, J_{2}\right)},
$$

3) for all $\left(m_{1}, J_{1}\right)$ and $\left(m_{2}, J_{2}\right)$,

$$
L_{n}^{\leqslant\left(m_{1}, J_{1}\right)} L_{n}^{\leqslant\left(m_{2}, J_{2}\right)} \subseteq L_{n}^{\leqslant\left(m_{1}, J_{1}\right) *\left(m_{2}, J_{2}\right)},
$$

4) the union of all such subspaces is equal to $L_{n}$

$$
\bigcup_{(m, J) \in \mathbb{Z} \times \mathscr{P}_{n}} L_{n}^{\leqslant(m, J)}=L_{n} .
$$

All of them follow directly from the definitions of $L_{n}^{\leqslant(m, J)}$ and $V_{n}(m, J)$. 
For $\left(m_{1}, J_{1}\right)$ and $\left(m_{2}, J_{2}\right)$ in $\mathbb{Z} \times \mathscr{P}_{n}$, we have

$$
L_{n}^{\leqslant\left(m_{1}, J_{1}\right)} L_{n}^{<\left(m_{2}, J_{2}\right)}+L_{n}^{<\left(m_{1}, J_{1}\right)} L_{n}^{\leqslant\left(m_{2}, J_{2}\right)} \subseteq L_{n}^{<\left(m_{1}, J_{1}\right) *\left(m_{2}, J_{2}\right)}
$$

and therefore the quotient spaces $L_{n}^{\leqslant(m, J)} / L_{n}^{<(m, J)}$ form the homogeneous components of the associated graded ring of $L_{n}$.

Theorem 2.6. With the filtration $\left\{L_{n}^{\leqslant(m, J)}:(m, J) \in \mathbb{Z} \times \mathscr{P}_{n}\right\}$ imposed on $L_{n}$, the direct sum decomposition of the associated graded ring

$$
\operatorname{gr} L_{n}=\bigoplus_{(m, J) \in \mathbb{Z} \times \mathscr{P}_{n}} L_{n}^{\leqslant(m, J)} / L_{n}^{<(m, J)}
$$

agrees with the decomposition of $g r L_{n}$ into its simple submodules over $\mathcal{U}_{n}$. In particular, for each $(m, J) \in \mathbb{Z} \times \mathscr{P}_{n}$, as a $\mathcal{U}_{n}$-module,

$$
L_{n}^{\leqslant(m, J)} / L_{n}^{<(m, J)} \cong W_{n}(m, J) .
$$

Proof. For an integer $m$ and a subset $J$ of $\{1,2, \ldots, n\}$ having $j$ elements, let us define

$$
S=V_{n}(m, J) \quad \text { and } \quad T=\sum_{J_{1} \subset J} V_{n}\left(m, J_{1}\right)+\sum_{m_{1}<m} V_{n}\left(m_{1}, J\right)
$$

where the first summation for $T$ is over all proper subsets $J_{1}$ of $J$, and the second summation is over all $m_{1}$ strictly less than $m$. Then, we have

$$
\begin{aligned}
S \cap T & =V_{n}(m, J) \cap\left(\sum_{J_{1} \subset J} V_{n}\left(m, J_{1}\right)+\sum_{m_{1}<m} V_{n}\left(m_{1}, J\right)\right) \\
& =V_{n}(m, J) \cap\left(\sum_{J_{1} \subset J} V_{n}\left(m, J_{1}\right)\right) \\
& =\sum_{J^{\prime}} V_{n}\left(m, J^{\prime}\right)
\end{aligned}
$$

where the last summation is over all subsets $J^{\prime}$ of $J$ having $j-1$ elements. Note that

$$
S+T=L_{n}^{\leqslant(m, J)} \text { and } \quad T=L_{n}^{<(m, J)}
$$

and then, by the usual module isomorphism theorem $(S+T) / T \cong S /(S \cap$ $T$ ), we obtain

$$
L_{n}^{\leqslant(m, J)} / L_{n}^{<(m, J)} \cong V_{n}(m, J) / \sum_{J^{\prime}} V_{n}\left(m, J^{\prime}\right)
$$


where the summation is over all subsets $J^{\prime}$ of $J$ having $j-1$ elements. Now, using the realization of $W_{n}(m, J)$ given in Lemma 2.3, we have

$$
L_{n}^{\leqslant(m, J)} / L_{n}^{<(m, J)} \cong W_{n}(m, J) .
$$

In $\S 6$, we will investigate the simple modules $W_{n}(m, J)$ in a more general setting.

\section{Modules $L_{n}^{\alpha}(m, j)$ and $V_{n}^{\alpha}(m, J)$}

In this section, generalizing $L_{n}(m, J)$ and $V_{n}(m, J)$ discussed in the previous section, we define some submodules of $L_{n}$ over the universal enveloping algebra $\mathcal{U}_{n}$ of $\mathfrak{g l}(n)$ parameterized by $\boldsymbol{\alpha}=\left(\alpha_{1}, \ldots, \alpha_{n}\right) \in \mathbb{C}^{n}$.

Notation 3.1. 1) For a finite set $S$, we will write $|S|$ for the cardinality of $S$.

2) For $\boldsymbol{\alpha}=\left(\alpha_{1}, \ldots, \alpha_{n}\right) \in \mathbb{C}^{n}$, we write $\boldsymbol{\alpha}[\ell]$ for the $\ell$ th component $\alpha_{\ell}$ of $\boldsymbol{\alpha}$. Then, for $\boldsymbol{\alpha}, \boldsymbol{\beta} \in \mathbb{C}^{n}$, we let $\boldsymbol{\alpha} \pm \boldsymbol{\beta}$ be the elements in $\mathbb{C}^{n}$ such that

$$
(\boldsymbol{\alpha} \pm \boldsymbol{\beta})[\ell]=\boldsymbol{\alpha}[\ell] \pm \boldsymbol{\beta}[\ell] \quad \text { for } 1 \leqslant \ell \leqslant n .
$$

3) We write $\mathbf{e}_{j}$ for the element in $\mathbb{Z}^{n}$ whose $j$ th entry is one and all the other entries are zero.

$$
\mathbf{e}_{j}[\ell]= \begin{cases}1 & \text { if } \ell=j, \\ 0 & \text { otherwise. }\end{cases}
$$

4) For $\mathbf{k} \in \mathbb{Z}^{n}$, let $\mathbf{x}^{\mathbf{k}}$ be the monomial

$$
\mathbf{x}^{\mathbf{k}}=x_{1}^{\mathbf{k}[1]} x_{2}^{\mathbf{k}[2]} \cdots x_{n}^{\mathbf{k}[n]}
$$

in $L_{n}$. In this setting, we denote the negative part of $\mathbf{k}$ by

$$
\mathbf{k}^{n e g}=\{\ell: \mathbf{k}[\ell]<0\} .
$$

We let $E_{a b} \in \mathfrak{g l}(n)$ be the $n \times n$ matrix with one in $(a, b)$ and zero elsewhere.

Definition 3.2. For each $\boldsymbol{\alpha} \in \mathbb{C}^{n}, E_{a b}$ acts on the monomials $\mathbf{x}^{\mathbf{k}}$ in the algebra $L_{n}$ of Laurent polynomials as

$$
E_{a b} \cdot \mathbf{x}^{\mathbf{k}}=(\mathbf{k}[b]+\boldsymbol{\alpha}[b]) \mathbf{x}^{\mathbf{k}+\mathbf{e}_{a}-\mathbf{e}_{b}} \quad \text { for } 1 \leqslant a, b \leqslant n .
$$

With this action, the space $L_{n}$ gives rise to a $\mathcal{U}_{n}$-module, which we will denote by $L_{n}^{\alpha}$, and for $f \in L_{n}$ we write $\langle f\rangle$ for the cyclic submodule of $L_{n}^{\alpha}$ generated by $f$. 
Informally, we may think of the above action as

$$
E_{a b} \cdot f=\mathbf{x}^{-\alpha} x_{a} \frac{\partial}{\partial x_{b}} \mathbf{x}^{\alpha} f \quad \text { for } f \in L_{n}
$$

and then the action in the definition can be considered a generalization of the action (1.2) of $\mathfrak{g l}(n)$ on the polynomial ring which provides all the finite dimensional representations of $\mathfrak{g l}(n)$ labeled by Young diagrams with single rows. See Theorem 5.4 (2).

Lemma 3.3. For $\boldsymbol{\alpha}$ and $\boldsymbol{\beta} \in \mathbb{C}^{n}$, if $\boldsymbol{\alpha}-\boldsymbol{\beta} \in \mathbb{Z}^{n}$ then, as a $\mathcal{U}_{n}$-module, $L_{n}^{\boldsymbol{\alpha}}$ is isomorphic to $L_{n}^{\boldsymbol{\beta}}$.

Proof. It is enough to show that the linear map $\psi$ from $L_{n}$ to $L_{n}$ sending $\mathbf{x}^{\mathbf{k}}$ to $\mathbf{x}^{\mathbf{k}+\boldsymbol{\alpha}-\boldsymbol{\beta}}$ for $\mathbf{k} \in \mathbb{Z}^{n}$ gives a $\mathcal{U}_{n}$-module map from $L_{n}^{\boldsymbol{\alpha}}$ to $L_{n}^{\boldsymbol{\beta}}$. It follows from

$$
\begin{aligned}
\psi\left(E_{a b} \cdot \mathbf{x}^{\mathbf{k}}\right) & =(\mathbf{k}[b]+\boldsymbol{\alpha}[b]) \mathbf{x}^{\mathbf{k}+\mathbf{e}_{a}-\mathbf{e}_{b}} \times \mathbf{x}^{\boldsymbol{\alpha}-\boldsymbol{\beta}} \\
& =\{(\mathbf{k}+\boldsymbol{\alpha}-\boldsymbol{\beta})[b]+\boldsymbol{\beta}[b]\} \mathbf{x}^{(\mathbf{k}+\boldsymbol{\alpha}-\boldsymbol{\beta})+\mathbf{e}_{a}-\mathbf{e}_{b}} \\
& =E_{a b} \cdot \mathbf{x}^{(\mathbf{k}+\boldsymbol{\alpha}-\boldsymbol{\beta})}=E_{a b} \cdot \psi\left(\mathbf{x}^{\mathbf{k}}\right)
\end{aligned}
$$

for all $1 \leqslant a, b \leqslant n$.

With this lemma, we can focus on the following choice of $\boldsymbol{\alpha}$.

Notation 3.4. Once and for all, we fix $\alpha \in \mathbb{C}^{n}$ the entries of whose real parts satisfy

$$
0 \leqslant \operatorname{Re}(\boldsymbol{\alpha}[\ell])<1 \quad \text { for all } 1 \leqslant \ell \leqslant n,
$$

and the following subset of $\{1,2, \ldots, n\}$

$$
I_{\boldsymbol{\alpha}}=\{\ell: \boldsymbol{\alpha}[\ell]=0\} .
$$

With this choice of $\boldsymbol{\alpha}$, we note that for $\mathbf{k} \in \mathbb{Z}^{n}$,

$$
\mathbf{k}[\ell]+\boldsymbol{\alpha}[\ell]=0 \quad \text { for some } \ell
$$

only when $\mathbf{k}[\ell]=\boldsymbol{\alpha}[\ell]=0$.

Definition 3.5 (Submodules $L_{n}^{\boldsymbol{\alpha}}(m, j)$ and $\left.V_{n}^{\boldsymbol{\alpha}}(m, J)\right)$.

1) For integers $m$ and $j$ with $0 \leqslant j \leqslant\left|I_{\boldsymbol{\alpha}}\right|$, we let $L_{n}^{\boldsymbol{\alpha}}(m, j)$ be the subspace of $L_{n}^{\alpha}$ spanned by all the monomials $\mathbf{x}^{\mathbf{k}}$ such that

$$
\sum_{\ell=1}^{n} \mathbf{k}[\ell]=m \quad \text { and } \quad\left|\mathbf{k}^{n e g} \cap I_{\boldsymbol{\alpha}}\right| \leqslant j .
$$


2) For a subset $J$ of $I_{\boldsymbol{\alpha}}$ with $|J|=j$, we let $V_{n}^{\boldsymbol{\alpha}}(m, J)$ be the subspace of $L_{n}^{\boldsymbol{\alpha}}(m, j)$ spanned by all the monomials $\mathbf{x}^{\mathbf{k}}$ in $L_{n}^{\boldsymbol{\alpha}}(m, j)$ such that

$$
\left(\mathbf{k}^{n e g} \cap I_{\boldsymbol{\alpha}}\right) \subseteq J .
$$

Example 3.6. Let $n=4, \boldsymbol{\alpha}=(0,1 / 2,0,0)$, and therefore $I_{\boldsymbol{\alpha}}=\{1,3,4\}$.

$1)$ Let $j=2$. From the condition $\left|\mathbf{k}^{\text {neg }} \cap\{1,3,4\}\right| \leqslant 2$, the space $L_{n}^{\alpha}(m, j)$ is spanned by all the monomials $\mathbf{x}^{\mathbf{k}}=x_{1}^{k_{1}} x_{2}^{k_{2}} x_{3}^{k_{3}} x_{4}^{k_{4}}$ in $L_{n}$ such that

i) $k_{1}+k_{2}+k_{3}+k_{4}=m$ and ii) $k_{1} \geqslant 0$ or $k_{3} \geqslant 0$ or $k_{4} \geqslant 0$.

2) Let $J=\{1,4\}$. From the condition $\left(\mathbf{k}^{\text {neg }} \cap\{1,3,4\}\right) \subset\{1,4\}$, $V_{n}^{\boldsymbol{\alpha}}(m, J)$ is the subspace of $L_{n}^{\boldsymbol{\alpha}}(m, j)$ spanned by all the monomials of degree $m$ with $k_{3} \geqslant 0$.

See more examples in Example 4.2. The following is easy to check.

Lemma 3.7. If $J=\varnothing$ (therefore $j=0$ ) or $J=I_{\alpha}$ (therefore $j=\left|I_{\alpha}\right|$ ), then

$$
V_{n}^{\alpha}(m, J)=L_{n}^{\alpha}(m, j) .
$$

From Definition 3.5, it immediately follows that

$$
L_{n}^{\alpha}(m, j-1) \subseteq L_{n}^{\alpha}(m, j) \quad \text { and } \quad L_{n}^{\boldsymbol{\alpha}}(m, j)=\sum_{J:|J|=j} V_{n}^{\boldsymbol{\alpha}}(m, J)
$$

where the summation runs over all subsets $J$ of $I_{\boldsymbol{\alpha}}$ with $|J|=j$. Also, for two subsets $J_{1}$ and $J_{2}$ of $I_{\boldsymbol{\alpha}}$, we have

$$
\begin{aligned}
& V_{n}^{\boldsymbol{\alpha}}\left(m, J_{1}\right) \subset V_{n}^{\boldsymbol{\alpha}}\left(m, J_{2}\right) \quad \text { for } J_{1} \subset J_{2} ; \\
& V_{n}^{\boldsymbol{\alpha}}\left(m, J_{1} \cap J_{2}\right)=V_{n}^{\boldsymbol{\alpha}}\left(m, J_{1}\right) \cap V_{n}^{\boldsymbol{\alpha}}\left(m, J_{2}\right) .
\end{aligned}
$$

Now we show that $V_{n}^{\alpha}(m, J)$ and $L_{n}^{\alpha}(m, j)$ are indeed modules over $\mathcal{U}_{n}$.

Proposition 3.8. $V_{n}^{\boldsymbol{\alpha}}(m, J)$ and $L_{n}^{\boldsymbol{\alpha}}(m, j)$ are $\mathcal{U}_{n}$-submodules of $L_{n}^{\boldsymbol{\alpha}}$.

Proof. For a monomial $\mathbf{x}^{\mathbf{p}} \in V_{n}^{\boldsymbol{\alpha}}(m, J)$, we need to show

$$
E_{a b} \cdot \mathbf{x}^{\mathbf{p}}=(\mathbf{p}[b]+\boldsymbol{\alpha}[b]) \mathbf{x}^{\mathbf{p}+\mathbf{e}_{a}-\mathbf{e}_{b}}
$$

are in $V_{n}^{\boldsymbol{\alpha}}(m, J)$ for all $1 \leqslant a, b \leqslant n$. Since the action of $E_{a b}$ preserves the degree of monomials, writing $\mathbf{q}=\left(\mathbf{p}+\mathbf{e}_{a}-\mathbf{e}_{b}\right)$, it is enough to show 
that for $a \neq b$ if $\left(\mathbf{p}^{n e g} \cap I_{\boldsymbol{\alpha}}\right) \subseteq J$, then $\left(\mathbf{q}^{\text {neg }} \cap I_{\boldsymbol{\alpha}}\right) \subseteq J$ or the coefficient $(\mathbf{p}[b]+\boldsymbol{\alpha}[b])$ is zero.

For this, because the only element which is not in $\mathbf{p}^{\text {neg }}$ but can possibly appear in $\mathbf{q}^{\text {neg }}$ is $b$, it is enough to consider the case

$$
b \notin \mathbf{p}^{n e g} \cap I_{\alpha} \quad \text { and } \quad b \in \mathbf{q}^{n e g} \cap I_{\alpha} .
$$

This happens only when $\mathbf{p}[b]=0$ and in this case, since $b \in I_{\boldsymbol{\alpha}}$, we have $\boldsymbol{\alpha}[b]=0$. Therefore, the coefficient $(\mathbf{p}[b]+\boldsymbol{\alpha}[b])$ is zero. Consequently, we have $E_{a b} \cdot \mathbf{x}^{\mathbf{p}} \in V_{n}^{\boldsymbol{\alpha}}(m, J)$ for all $1 \leqslant a, b \leqslant n$, and $V_{n}^{\boldsymbol{\alpha}}(m, J)$ is a submodule of $L_{n}^{\boldsymbol{\alpha}}$. Now from (3.2), $L_{n}^{\boldsymbol{\alpha}}(m, j)$ is a submodule of $L_{n}^{\boldsymbol{\alpha}}$.

\section{Structure of $V_{n}^{\alpha}(m, J)$}

In this section, we investigate the structure of $V_{n}^{\alpha}(m, J)$. We first give a technical lemma.

Lemma 4.1. For two distinct monomials $\mathbf{x}^{\mathbf{p}}$ and $\mathrm{x}^{\mathbf{q}}$ in $L_{n}^{\alpha}(m, j)$ such that

$$
\left(\mathbf{q}^{n e g} \cap I_{\boldsymbol{\alpha}}\right) \subseteq\left(\mathbf{p}^{n e g} \cap I_{\boldsymbol{\alpha}}\right),
$$

there exists $X \in \mathcal{U}_{n}$ such that $X \cdot \mathrm{x}^{\mathbf{p}}=\mathrm{x}^{\mathrm{q}}$.

Proof. For simplicity, we let $\mathbf{p}[\ell]=p_{\ell}, \mathbf{q}[\ell]=q_{\ell}$, and $\boldsymbol{\alpha}[\ell]=\alpha_{\ell}$ for all $\ell$. Consider the difference $\mathbf{p}-\mathbf{q}=\left(p_{1}-q_{1}, \ldots, p_{n}-q_{n}\right) \in \mathbb{Z}^{n}$. Since $\mathbf{x}^{\mathbf{p}}$ and $\mathrm{x}^{\mathbf{q}}$ have the same degree $m$, we have $\sum_{\ell=1}^{n}\left(p_{\ell}-q_{\ell}\right)=0$ and therefore we can separate the positive and negative parts of $\mathbf{p}-\mathbf{q}$

$$
r=\sum_{\ell: p_{\ell}-q_{\ell}>0}\left(p_{\ell}-q_{\ell}\right)=\sum_{\ell: p_{\ell}-q_{\ell}<0}\left(q_{\ell}-p_{\ell}\right) .
$$

With Notation 3.1 , we define $1 \leqslant s_{1} \leqslant s_{2} \leqslant \cdots \leqslant s_{r} \leqslant n$ and $1 \leqslant t_{1} \leqslant$ $t_{2} \leqslant \cdots \leqslant t_{r} \leqslant n$ so that

$$
\sum_{k=1}^{r} \mathbf{e}_{s_{k}}=\sum_{\ell: p_{\ell}-q_{\ell}>0}\left(p_{\ell}-q_{\ell}\right) \mathbf{e}_{\ell} \quad \text { and } \quad \sum_{k=1}^{r} \mathbf{e}_{t_{k}}=\sum_{\ell: p_{\ell}-q_{\ell}<0}\left(q_{\ell}-p_{\ell}\right) \mathbf{e}_{\ell}
$$

where the summations are over $\ell$ such that $p_{\ell}-q_{\ell}>0$ and $p_{\ell}-q_{\ell}<0$ respectively. 
Setting $\mathbf{p}_{0}=\mathbf{p}$ and $\mathbf{p}_{k}=\mathbf{p}_{k-1}+\mathbf{e}_{t_{k}}-\mathbf{e}_{s_{k}}$ for $1 \leqslant k \leqslant r$, we have $E_{t_{k} s_{k}} \cdot \mathbf{x}^{\mathbf{p}_{k-1}}=w_{k} \mathbf{x}^{\mathbf{p}_{k}}$. Furthermore, from

$$
\begin{aligned}
\mathbf{p}_{r} & =\mathbf{p}_{0}+\sum_{k=1}^{r}\left(\mathbf{e}_{t_{k}}-\mathbf{e}_{s_{k}}\right) \\
& =\mathbf{p}_{0}+\sum_{\ell: p_{\ell}-q_{\ell}<0}\left(q_{\ell}-p_{\ell}\right) \mathbf{e}_{\ell}-\sum_{\ell: p_{\ell}-q_{\ell}>0}\left(p_{\ell}-q_{\ell}\right) \mathbf{e}_{\ell} \\
& =\mathbf{p}_{0}+(\mathbf{q}-\mathbf{p})=\mathbf{q},
\end{aligned}
$$

by setting $Y=\prod_{k=1}^{r} E_{t_{k} s_{k}} \in \mathcal{U}_{n}$, we obtain

$$
\begin{aligned}
Y \cdot \mathbf{x}^{\mathbf{p}} & =\mathbf{x}^{\mathbf{p}}\left(w_{1} x_{t_{1}} x_{s_{1}}^{-1}\right)\left(w_{2} x_{t_{2}} x_{s_{2}}^{-1}\right) \cdots\left(w_{r} x_{t_{r}} x_{s_{r}}^{-1}\right) \\
& =\left(\prod_{k=1}^{r} w_{k}\right) \mathbf{x}^{\mathbf{p}_{\mathbf{x}}} \mathbf{q}-\mathbf{p}=\left(\prod_{k=1}^{r} w_{k}\right) \mathbf{x}^{\mathbf{q}}
\end{aligned}
$$

where the coefficient is

$$
\prod_{k=1}^{r} w_{k}=\prod_{\ell: p_{\ell}-q_{\ell}>0}\left(p_{\ell}+\alpha_{\ell}\right)\left(p_{\ell}-1+\alpha_{\ell}\right) \cdots\left(p_{\ell}-\left(p_{\ell}-q_{\ell}-1\right)+\alpha_{\ell}\right) .
$$

For each $\ell$ in the above product, if $\ell \notin I_{\boldsymbol{\alpha}}$ then $\alpha_{\ell} \neq 0$, and therefore the corresponding factor is not zero by (3.1). Now let $\ell \in I_{\boldsymbol{\alpha}}$ and therefore $\alpha_{\ell}=0$. To derive a contradiction, suppose $\left(p_{\ell}-c\right)$ in the $\ell$ th factor of the above product is zero for some $0 \leqslant c \leqslant\left(p_{\ell}-q_{\ell}-1\right)$. Then, from $p_{\ell}=c$, we have

$$
0 \leqslant p_{\ell} \leqslant\left(p_{\ell}-q_{\ell}-1\right)
$$

and therefore $q_{\ell} \leqslant-1$. On the other hand, from the given hypothesis $\left(\mathbf{q}^{\text {neg }} \cap I_{\boldsymbol{\alpha}}\right) \subseteq\left(\mathbf{p}^{\text {neg }} \cap I_{\boldsymbol{\alpha}}\right)$, we know that $p_{\ell}<0$ whenever $q_{\ell}<0$, which contradicts to (4.1). Therefore, we have $\prod_{k=1}^{r} w_{k} \neq 0$ and with the element

$$
X=\prod_{k=1}^{r} w_{k}^{-1} E_{t_{k} s_{k}}
$$

we see that $X \cdot \mathbf{x}^{\mathbf{p}}=\mathbf{x}^{\mathbf{q}}$.

Example 4.2. 1) Let $\boldsymbol{\alpha}=(1 / 2, i, 0), I_{\boldsymbol{\alpha}}=\{3\}$, and $J=\varnothing$. If $m=4$ then from the condition

$$
\mathbf{k}^{n e g} \cap\{3\} \subseteq \varnothing,
$$

$V_{n}^{\boldsymbol{\alpha}}(m, J)$ is spanned by all the monomials $x_{1}^{k_{1}} x_{2}^{k_{2}} x_{3}^{k_{3}}$ of degree 4 with $k_{3} \geqslant 0$. 
Note that $\mathbf{x}^{\mathbf{p}}=x_{1}^{4}$ and $\mathbf{x}^{\mathbf{q}}=x_{1} x_{2}^{-2} x_{3}^{5}$ in $V_{n}^{\boldsymbol{\alpha}}(m, J)$ satisfy the condition in Lemma 4.1. From

$$
\mathbf{p}-\mathbf{q}=(3,2,-5)=(3,2,0)+(0,0,-5)
$$

we define the element $Y=E_{31} \cdot E_{31} \cdot E_{31} \cdot E_{32} \cdot E_{32} \in \mathcal{U}_{n}$ to obtain

$$
Y \cdot \mathbf{x}^{\mathbf{p}}=(2+1 / 2)(3+1 / 2)(4+1 / 2)(-1+i)(0+i) \mathbf{x}^{\mathbf{q}} .
$$

Therefore there is $X \in \mathcal{U}_{n}$ such that $X \cdot \mathbf{x}^{\mathbf{p}}=\mathrm{x}^{\mathbf{q}}$.

2) Let $\alpha=(0,0,0), I_{\boldsymbol{\alpha}}=\{1,2,3\}$, and $J=\{1,3\}$. If $m=-2$ then from the condition

$$
\mathbf{k}^{\text {neg }} \cap\{1,2,3\} \subseteq\{1,3\},
$$

$V_{n}^{\alpha}(m, J)$ is spanned by all the monomials $x_{1}^{k_{1}} x_{2}^{k_{2}} x_{3}^{k_{3}}$ of degree -2 with $k_{2} \geqslant 0$.

Note that $\mathbf{x}^{\mathbf{p}}=x_{1}^{-1} x_{3}^{-1}$ and $\mathbf{x}^{\mathbf{q}}=x_{1} x_{2}^{2} x_{3}^{-5}$ in $V_{n}^{\boldsymbol{\alpha}}(m, J)$ satisfy the condition in Lemma 4.1. From

$$
\mathbf{p}-\mathbf{q}=(-2,-2,4)=(0,0,4)+(-2,-2,0)
$$

we define $Y=E_{13} \cdot E_{13} \cdot E_{23} \cdot E_{23} \in \mathcal{U}_{n}$ to obtain

$$
Y \cdot \mathbf{x}^{\mathbf{p}}=(-4+0)(-3+0)(-2+0)(-1+0) \mathbf{x}^{\mathbf{q}} .
$$

Therefore, there is $X \in \mathcal{U}_{n}$ such that $X \cdot \mathrm{x}^{\mathbf{p}}=\mathrm{x}^{\mathbf{q}}$.

Now we investigate the structure of $V_{n}^{\boldsymbol{\alpha}}(m, J)$ for $\boldsymbol{\alpha} \neq \mathbf{0}$.

Theorem 4.3 (Structure of $V_{n}^{\alpha}(m, J)$ with nonzero $\boldsymbol{\alpha}$ ).

Let $\boldsymbol{\alpha} \neq \mathbf{0}$ and $J=\left\{\ell_{1}, \ldots, \ell_{j}\right\} \subseteq I_{\boldsymbol{\alpha}}$. Then, $V_{n}^{\boldsymbol{\alpha}}(m, J)$ is the cyclic submodule of $L_{n}^{\alpha}$ generated by

$$
\mathbf{x}_{J, t}=x_{\ell_{1}}^{-1} x_{\ell_{2}}^{-1} \cdots x_{\ell_{j}}^{-1} x_{t}^{m+j} \quad \text { for some } t \in\{1,2, \ldots, n\} \backslash I_{\boldsymbol{\alpha}} .
$$

Proof. Since $\boldsymbol{\alpha} \neq \mathbf{0}$, there exists $t$ such that $\boldsymbol{\alpha}[t] \neq 0$. Write $\mathbf{x}^{\mathbf{p}}$ for $\mathbf{x}_{J, t}$ and let $\mathbf{x}^{\mathbf{q}}$ be an arbitrary monomial in $V_{n}^{\boldsymbol{\alpha}}(m, J)$. Since $\left(\mathbf{q}^{\text {neg }} \cap I_{\boldsymbol{\alpha}}\right) \subseteq$ $\left(\mathbf{p}^{n e g} \cap I_{\boldsymbol{\alpha}}\right)=J$, we can apply Lemma 4.1 to obtain $X \in \mathcal{U}_{n}$ such that $X \cdot \mathbf{x}^{\mathbf{p}}=\mathbf{x}^{\mathbf{q}}$. Therefore, $\mathbf{x}^{\mathbf{p}}$ generates the module $V_{n}^{\alpha}(m, J)$.

Next we consider the other cases with $\boldsymbol{\alpha}=\mathbf{0}$. Let us fix

$$
J=\left\{\ell_{1}, \ldots, \ell_{j}\right\} \subseteq I_{\alpha}=\{1,2, \ldots, n\} .
$$


Theorem 4.4 (Structure of $V_{n}^{\mathbf{0}}(m, J)$ with nonnegative degree $m$ ).

1) If $m \geqslant 0$ and $0 \leqslant j \leqslant n-1$, then $V_{n}^{\mathbf{0}}(m, J)$ is the cyclic submodule of $L_{n}^{\mathbf{0}}(m, j)$ generated by

$$
\mathbf{x}_{J, t}=x_{\ell_{1}}^{-1} x_{\ell_{2}}^{-1} \cdots x_{\ell_{j}}^{-1} x_{t}^{m+j} \quad \text { for some } t \in\{1,2, \ldots, n\} \backslash J .
$$

2) If $m \geqslant 0$ and $j=n$ (therefore, $J=\{1,2, \ldots, n\}$ ), then

$$
\begin{aligned}
V_{n}^{\mathbf{0}}(m,\{1,2, \ldots, n\}) & =L_{n}^{\mathbf{0}}(m, n)=L_{n}^{\mathbf{0}}(m, n-1) \\
& =\sum_{J^{\prime}} V_{n}^{\mathbf{0}}\left(m, J^{\prime}\right)
\end{aligned}
$$

where the summation is over all the subsets $J^{\prime}$ of $\{1,2, \ldots, n\}$ with $\left|J^{\prime}\right|=n-1$.

Proof. For Statement (1), we first note that $\mathbf{x}_{J, t} \in V_{n}^{\mathbf{0}}(m, J)$. Write $\mathbf{x}^{\mathbf{p}}$ for $\mathbf{x}_{J, t}$ and let $\mathbf{x}^{\mathbf{q}}$ be an arbitrary monomial in $V_{n}^{\mathbf{0}}(m, J)$. Since $\mathbf{q}^{\text {neg }} \subseteq$ $\mathbf{p}^{n e g}=J$, applying Lemma 4.1, we see that there exists $X \in \mathcal{U}_{n}$ such that $X \cdot \mathbf{x}^{\mathbf{p}}=\mathbf{x}^{\mathbf{q}}$. Therefore, $\mathbf{x}^{\mathbf{q}}$ belongs to the module generated by $\mathbf{x}_{J, t}$ and we have $V_{n}^{\mathbf{0}}(m, J)=\left\langle\mathbf{x}_{J, t}\right\rangle$.

For Statement (2), if $x_{1}^{k_{1}} x_{2}^{k_{2}} \cdots x_{n}^{k_{n}}$ is a monomial in $L_{n}^{\mathbf{0}}(m, n)$, then not all $k_{j}$ 's can be negative, because the degree $m$ is nonnegative. Therefore, $L_{n}^{\mathbf{0}}(m, n)=L_{n}^{\mathbf{0}}(m, n-1)$. The other equalities follow from Lemma 3.7 and (3.2).

Theorem 4.5 (Structure of $V_{n}^{\mathbf{0}}(-m, J)$ with negative degree $-m$ ).

Let $1 \leqslant m<n$.

1a) If $j=0$ (therefore, $J=\varnothing$ ), then

$$
V_{n}^{\mathbf{0}}(-m, \varnothing)=L_{n}^{\mathbf{0}}(-m, 0)=\{0\}
$$

1b) If $1 \leqslant j \leqslant m$, then $V_{n}^{\mathbf{0}}(-m, J)$ is the cyclic submodule of $L_{n}^{\mathbf{0}}(-m, j)$ generated by

$$
\mathbf{x}_{J}=x_{\ell_{1}}^{-1} x_{\ell_{2}}^{-1} \cdots x_{\ell_{j-1}}^{-1} x_{\ell_{j}}^{j-1-m}
$$

1c) If $m+1 \leqslant j \leqslant n-1$, then $V_{n}^{\mathbf{0}}(-m, J)$ is the cyclic submodule of $L_{n}^{\mathbf{0}}(-m, j)$ generated by

$$
\mathbf{x}_{J, t}=x_{\ell_{1}}^{-1} x_{\ell_{2}}^{-1} \cdots x_{\ell_{j-1}}^{-1} x_{\ell_{j}}^{-1} x_{t}^{j-m} \quad \text { for some } t \in\{1,2, \ldots, n\} \backslash J .
$$


1d) If $j=n$ (therefore, $J=\{1,2, \ldots, n\}$ ), then

$$
\begin{aligned}
V_{n}^{\mathbf{0}}(-m,\{1,2, \ldots, n\}) & =L_{n}^{\mathbf{0}}(-m, n)=L_{n}^{\mathbf{0}}(-m, n-1) \\
& =\sum_{J^{\prime}} V_{n}^{\mathbf{0}}\left(-m, J^{\prime}\right)
\end{aligned}
$$

where the summation is over all $J^{\prime} \subset\{1,2, \ldots, n\}$ with $\left|J^{\prime}\right|=n-1$.

Now let $m \geqslant n$.

2a) If $j=0$ (therefore, $J=\varnothing$ ), then

$$
V_{n}^{\mathbf{0}}(-m, \varnothing)=L_{n}^{\mathbf{0}}(-m, 0)=\{0\} .
$$

2b) If $1 \leqslant j \leqslant n$, then $V_{n}^{\mathbf{0}}(-m, J)$ is the cyclic submodule of $L_{n}^{\mathbf{0}}(-m, j)$ generated by

$$
\mathbf{x}_{J}=x_{\ell_{1}}^{-1} x_{\ell_{2}}^{-1} \cdots x_{\ell_{j-1}}^{-1} x_{\ell_{j}}^{j-1-m} .
$$

Proof. Statements 1a) and 2a) follow directly from Definition 3.5. For Statement $1 \mathrm{~d})$, note that if $\mathbf{x}^{\mathbf{k}} \in L_{n}^{\mathbf{0}}(-m, n)$ then $\sum_{\ell} \mathbf{k}[\ell]=-m>-n$ and therefore there should be at least one $\ell$ with $\mathbf{k}[\ell] \geqslant 0$. This implies that $L_{n}^{\mathbf{0}}(-m, n)=L_{n}^{\mathbf{0}}(-m, n-1)$. Now the statements follows from Lemma 3.7 and (3.2). The other statements can be shown similarly to Theorem 4.3 and Theorem 4.4 (1).

Remark 4.6. Let $\mathbf{x}^{\mathbf{p}}$ be the generators $\mathrm{x}_{J}$ or $\mathbf{x}_{J, t}$ of the cyclic modules $V_{n}^{\boldsymbol{\alpha}}(m, J)$ given in Theorem 4.3, Theorem 4.4, and Theorem 4.5. We remark that these generators are not unique. This is because, when applying Lemma 4.1, if

$$
\left(\mathbf{q}^{n e g} \cap I_{\boldsymbol{\alpha}}\right)=\left(\mathbf{p}^{n e g} \cap I_{\boldsymbol{\alpha}}\right),
$$

then we can exchange the roles of $\mathbf{x}^{\mathbf{p}}$ and $\mathbf{x}^{\mathbf{q}}$. Therefore, every monomial $\mathbf{x}^{\mathbf{q}} \in V_{n}^{\boldsymbol{\alpha}}(m, J)$ satisfying (4.2) can also generate the module $V_{n}^{\boldsymbol{\alpha}}(m, J)$.

\section{Structure of $L_{n}^{\alpha}(m, j)$}

In this section, we investigate the structure of $L_{n}^{\boldsymbol{\alpha}}(m, j)$. Let us begin with another technical lemma.

Lemma 5.1. For an element $f=\sum_{i=1}^{r} c_{i} \mathbf{x}^{\mathbf{k}_{i}}$ of $L_{n}^{\alpha}$ with distinct monomials and nonzero coefficients, the cyclic module generated by $f$ includes the cyclic modules generated by the terms of $f$

$$
\left\langle\mathbf{x}^{\mathbf{k}_{i}}\right\rangle \subseteq\langle f\rangle \text { for all } i .
$$


Proof. We want to prove the statement by induction on the number $r$ of the terms of $f$. If $r=1$ then we have nothing to prove. Suppose $r \geqslant 2$. We first note that, since $\mathbf{x}^{\mathbf{k}_{i}}$ are distinct, they are weight vectors with different weights under the action of the Cartan subalgebra $\mathfrak{h}$ of $\mathfrak{g l}(n)$ spanned by $E_{a a}$ for $1 \leqslant a \leqslant n$. Let $w_{i}$ be the weight for the monomial $\mathbf{x}^{\mathbf{k}_{i}}$. Then, there is an element $H \in \mathfrak{h}$ such that

$$
g=w_{1}(H) f-H \cdot f=\sum_{i: w_{i}(H) \neq w_{1}(H)}\left(w_{1}(H)-w_{i}(H)\right) c_{i} \mathbf{x}^{\mathbf{k}_{i}}
$$

is a non-zero element in $\langle f\rangle$. Also, since the number of terms in $g$ is less than $r$, by the induction hypothesis the cyclic modules generated by the terms of $g$ are included in $\langle g\rangle$. This shows that for $i$ with $w_{i}(H) \neq w_{1}(H)$ we have $\left\langle\mathbf{x}^{\mathbf{k}_{i}}\right\rangle \subseteq\langle f\rangle$.

Now we note that

$$
h=f-\sum_{i: w_{i}(H) \neq w_{1}(H)} c_{i} \mathbf{x}^{\mathbf{k}_{i}}=\sum_{i: w_{i}(H)=w_{1}(H)} c_{i} \mathbf{x}^{\mathbf{k}_{i}} .
$$

is a nonzero element in $\langle f\rangle$. Since the number of the terms of $h$ is less than $r$, again by the induction hypothesis, the cyclic modules generated by the terms of $h$ are included in $\langle h\rangle$. Therefore, $\langle f\rangle$ contains $\left\langle\mathbf{x}^{\mathbf{k}_{i}}\right\rangle$ for $i$ with $w_{i}(H)=w_{1}(H)$ as well.

As an immediate consequence of the above lemma, we obtain the following result for the special case of Theorem 4.5 with $J=\{\ell\}$ for $1 \leqslant \ell \leqslant n$.

Proposition 5.2. For $m \geqslant 1$ and $1 \leqslant \ell \leqslant n, V_{n}^{\mathbf{0}}(-m,\{\ell\})$ is a simple submodule of $L_{n}^{\mathbf{0}}(-m, 1)$ generated by $x_{\ell}^{-m}$.

Proof. From Theorem 4.5 1b) and 2b),

$$
V_{n}^{\mathbf{0}}(-m, J)=\left\langle x_{\ell}^{-m}\right\rangle \subseteq L_{n}^{\mathbf{0}}(-m, 1) .
$$

For a nonzero $f \in V_{n}^{\mathbf{0}}(-m, J)$, writing $f=\sum_{i} c_{i} \mathbf{x}^{\mathbf{k}_{i}}$, let us consider the submodule of $V_{n}^{\mathbf{0}}(-m, J)$ generated by $f$. By Lemma 5.1, it contains the cyclic submodules generated by $\mathbf{x}^{\mathbf{k}_{i}}$.

$$
\left\langle\mathbf{x}^{\mathbf{k}_{i}}\right\rangle \subseteq\langle f\rangle \subseteq V_{n}^{\mathbf{0}}(-m, J) .
$$

On the other hand, for each $i$, since $\left(\mathbf{k}_{i}^{n e g} \cap\{1,2, \ldots, n\}\right) \subseteq\{\ell\}$ and the degree of $\mathbf{x}^{\mathbf{k}_{i}}$ should be $-m<0$, we have $\mathbf{k}_{i}[\ell]<0$ and $\mathbf{k}_{i}\left[\ell^{\prime}\right] \geqslant 0$ for $\ell^{\prime} \neq \ell$. By Remark 4.6, each of these monomials can generate the whole module $V_{n}^{\mathbf{0}}(-m, J)$. Therefore, we have $\langle f\rangle=V_{n}^{\mathbf{0}}(-m, J)$ and conclude that $V_{n}^{\mathbf{0}}(-m,\{\ell\})$ has no nonzero proper submodules. 
Now, we investigate the structure of $L_{n}^{\boldsymbol{\alpha}}(m, j)$ for $\boldsymbol{\alpha} \neq \mathbf{0}$.

Theorem 5.3 (Structure of $L_{n}^{\boldsymbol{\alpha}}(m, j)$ with nonzero $\boldsymbol{\alpha}$ ).

1) If $\boldsymbol{\alpha} \neq \mathbf{0}$ and $0 \leqslant j \leqslant\left|I_{\boldsymbol{\alpha}}\right|$, then $L_{n}^{\boldsymbol{\alpha}}(m, j)$ is indecomposable.

2) In particular, if $\boldsymbol{\alpha} \neq \mathbf{0}$ and $j=0$, then $L_{n}^{\boldsymbol{\alpha}}(m, 0)=V_{n}^{\boldsymbol{\alpha}}(m, \varnothing)$ is a nonzero simple module over $\mathcal{U}_{n}$.

Proof. For Statement (2), from Lemma 3.7, $L_{n}^{\boldsymbol{\alpha}}(m, 0)=V_{n}^{\boldsymbol{\alpha}}(m, \varnothing)$, and by Theorem 4.3 it is generated by $\mathbf{x}_{\varnothing, t}=x_{t}^{m}$ for some $t \in\{1,2, \ldots, n\} \backslash I_{\boldsymbol{\alpha}}$. Let $f$ be a nonzero element of $V_{n}^{\boldsymbol{\alpha}}(m, \varnothing)$, then we can write $f=\sum_{i=1}^{r} c_{i} \mathbf{x}^{\mathbf{k}_{i}}$ with nonzero coefficients such that $\mathbf{k}_{i}^{n e g} \cap I_{\boldsymbol{\alpha}}=\varnothing$ for all $i$. By Lemma 5.1, the monomials $\mathbf{x}^{\mathbf{k}_{i}}$ belong to $\langle f\rangle$. On the other hand, by Remark 4.6, each of these monomials generates $V_{n}^{\boldsymbol{\alpha}}(m, \varnothing)$. Therefore, $\langle f\rangle=V_{n}^{\boldsymbol{\alpha}}(m, \varnothing)$. This shows that $L_{n}^{\boldsymbol{\alpha}}(m, 0)=V_{n}^{\boldsymbol{\alpha}}(m, \varnothing)$ is simple.

For Statement (1), we will show that every nonzero submodule $M$ of $L_{n}^{\boldsymbol{\alpha}}(m, j)$ contains $V_{n}^{\boldsymbol{\alpha}}(m, \varnothing)$, which is nonzero by Statement (2). For a nonzero $f \in M$, let $c \mathbf{x}^{\mathbf{p}}$ be a nonzero term of $f$. Then, by Lemma 5.1, $\langle f\rangle$ includes $\left\langle\mathbf{x}^{\mathbf{p}}\right\rangle$. On the other hand, since every monomial $\mathbf{x}^{\mathbf{q}}=x_{t}^{m}$ for $t \notin I_{\boldsymbol{\alpha}}$ satisfies the condition

$$
\varnothing=\left(\mathbf{q}^{n e g} \cap I_{\boldsymbol{\alpha}}\right) \subseteq\left(\mathbf{p}^{n e g} \cap I_{\boldsymbol{\alpha}}\right),
$$

we can apply Lemma 4.1 to obtain $X \in \mathcal{U}_{n}$ such that $X \cdot \mathbf{x}^{\mathbf{p}}=\mathbf{x}^{\mathbf{q}}$. Therefore, we have $\mathbf{x}^{\mathbf{q}} \in\left\langle\mathbf{x}^{\mathbf{p}}\right\rangle \subseteq\langle f\rangle \subseteq M$, and therefore $V_{n}^{\boldsymbol{\alpha}}(m, \varnothing) \subseteq M$. This shows that $L_{n}^{\boldsymbol{\alpha}}(m, j)$ cannot be written as a direct sum of its proper nonzero submodules.

Next, we consider the other cases with $\boldsymbol{\alpha}=\mathbf{0}$.

Theorem 5.4 (Structure of $L_{n}^{\mathbf{0}}(m, j)$ with nonnegative degree $m$ ).

1) If $m \geqslant 0$ and $1 \leqslant j \leqslant n$, then $L_{n}^{\mathbf{0}}(m, j)$ is indecomposable.

2) In particular, if $m \geqslant 0$ and $j=0$, then $L_{n}^{\mathbf{0}}(m, 0)=V_{n}^{\mathbf{0}}(m, \varnothing)$ is the cyclic module generated by

$$
\mathbf{x}_{\varnothing, 1}=x_{1}^{m}
$$

It is a finite dimensional simple module over $\mathfrak{g l}(n)$ of dimension $(m+1)$.

Proof. For Statement (2), observe that for $m \geqslant 0, L_{n}^{\mathbf{0}}(m, 0)$ is the space of homogeneous polynomials of degree $m$,

$$
L_{n}^{\mathbf{0}}(m, 0) \cong \operatorname{Sym}^{m}\left(\mathbb{C}^{n}\right)
$$


and that $x_{1}^{m}$ is the highest weight vector with respect to the standard Borel subalgebra of upper triangular matrices in $\mathfrak{g l}(n)$.

For Statement (1), if $j=n$, then $L_{n}^{\mathbf{0}}(m, n)=L_{n}^{\mathbf{0}}(m, n-1)$ by Theorem 4.4 (2) and therefore we can assume $0 \leqslant j \leqslant n-1$. This case can be shown similarly to Theorem 5.3 (1).

Theorem 5.5 (Structure of $L_{n}^{0}(-m, j)$ with negative degree $-m$ ).

1) If $m \geqslant 1$ and $j=0$, then $L_{n}^{\mathbf{0}}(-m, j)=V_{n}^{\mathbf{0}}(-m, \varnothing)=\{0\}$.

2) If $m \geqslant 1$ and $j=1$, then $L_{n}^{\mathbf{0}}(-m, j)$ decomposes into simple submodules

$$
L_{n}^{\mathbf{0}}(-m, 1)=\bigoplus_{\ell=1}^{n} V_{n}^{\mathbf{0}}(-m,\{\ell\}) .
$$

3) If $m \geqslant 1$ and $2 \leqslant j \leqslant n$, then $L_{n}^{\mathbf{0}}(-m, j)$ is indecomposable.

Proof. Statement (1) is straightforward to check. For Statement (2), from (3.2) and Theorem $4.51 \mathrm{~b})$ and $2 \mathrm{~b})$, the module $L_{n}^{\mathbf{0}}(-m, 1)$ is the sum of the cyclic modules $V_{n}^{\mathbf{0}}(-m,\{\ell\})=\left\langle x_{\ell}^{-m}\right\rangle$, which are simple by Proposition 5.2. Also, for $\ell \neq \ell^{\prime}$, by (3.3),

$$
V_{n}^{\mathbf{0}}(-m,\{\ell\}) \cap V_{n}^{\mathbf{0}}\left(-m,\left\{\ell^{\prime}\right\}\right)=V_{n}^{\mathbf{0}}\left(-m,\{\ell\} \cap\left\{\ell^{\prime}\right\}\right)
$$

which is $V_{n}^{\mathbf{0}}(-m, \varnothing)=\{0\}$ by Statement (1). Hence we obtain the direct sum expression.

For Statement (3), we first consider the case $2 \leqslant j \leqslant n-1$. In order to derive a contradiction, suppose $L_{n}^{\mathbf{0}}(-m, j)=M \oplus N$ for some submodules $M$ and $N$. From $(3.2), L_{n}^{\mathbf{0}}(-m, j)$ is generated by the generators of $V_{n}^{\mathbf{0}}(-m, J)$ given in Theorem 4.5. We denote these generators $\mathbf{x}_{J}$ or $\mathbf{x}_{J, t}$ by $g_{J}$. For each of these monomials $g_{J} \in L_{n}^{\mathbf{0}}(-m, j)$, if $g_{J}=h_{1}+h_{2}$ with $h_{1} \in M$ and $h_{2} \in N$, then $g_{J}$ appears in $h_{1}$ or $h_{2}$. Therefore, by Lemma 5.1, $g_{J}$ belongs to $\left\langle h_{1}\right\rangle \subseteq M$ or $\left\langle h_{2}\right\rangle \subseteq N$.

If all these $g_{J}$ are in $M($ or $N)$, then $L_{n}^{\mathbf{0}}(-m, j)=M$ (or $\left.N\right)$. If some of them are in $M$ and some of them are in $N$, then we claim that there are $J$ and $J^{\prime}$ such that $g_{J}$ is an element in $M, g_{J^{\prime}}$ is an element in $N$, and $J \cap J^{\prime} \neq \varnothing$. Suppose there are not such $J$ and $J^{\prime}$. Then we can partition $\{1,2, \ldots, n\}$ into two nontrivial parts $S_{M}$ and $S_{N}$ with $n_{1}$ elements and $n_{2}$ elements such that we have the disjoint union

$$
\{J \subset\{1,2, \ldots, n\}:|J|=j\}=\left\{J \subset S_{M}:|J|=j\right\} \cup\left\{J \subset S_{N}:|J|=j\right\}
$$

where $J \subset S_{M}$ if and only if $g_{J}$ belongs to $M ; J \subset S_{N}$ if and only if $g_{J}$ belongs to $N$. Note that it contradicts to $\left(\begin{array}{c}n \\ j\end{array}\right)>\left(\begin{array}{c}n_{1} \\ j\end{array}\right)+\left(\begin{array}{c}n_{2} \\ j\end{array}\right)$ for 
$2 \leqslant j \leqslant n-1$. Therefore, we conclude that there are $J$ and $J^{\prime}$ such that $J \cap J^{\prime} \neq \varnothing$. Now from (3.3) we have

$$
V_{n}^{\mathbf{0}}\left(-m, J \cap J^{\prime}\right) \subset V_{n}^{\mathbf{0}}(-m, J) \subset M
$$

and

$$
V_{n}^{\mathbf{0}}\left(-m, J \cap J^{\prime}\right) \subset V_{n}^{\mathbf{0}}\left(-m, J^{\prime}\right) \subset N .
$$

Therefore, $V_{n}^{\mathbf{0}}\left(-m, J \cap J^{\prime}\right) \neq\{0\}$ and $M \cap N$ contains a non-trivial element. Hence, $L_{n}^{\mathbf{0}}(-m, j)$ is indecomposable.

Next, let us consider the case $j=n$. First, if $1 \leqslant m<n$ then from Theorem $4.51 \mathrm{~d})$ we have $L_{n}^{\mathbf{0}}(-m, n)=L_{n}^{\mathbf{0}}(-m, n-1)$ and therefore it goes back to the previous case. Second, if $m \geqslant n$ then $L_{n}^{\mathbf{0}}(-m, n)=$ $V_{n}^{\mathbf{0}}(-m,\{1,2, \ldots, n\})$ by Lemma 3.7 and its generator is $\mathbf{x}_{\{1,2, \ldots, n\}}=$ $x_{1}^{-1} \cdots x_{n-1}^{-1} x_{n}^{n-1-m}$ by Theorem $\left.4.52 \mathrm{~b}\right)$. If $L_{n}^{0}(-m, n)=M \oplus N$, then $\mathbf{x}_{\{1,2, \ldots, n\}}=h_{1}+h_{2}$ for some $h_{1} \in M$ and $h_{2} \in N$, and the monomial $\mathbf{x}_{\{1,2, \ldots, n\}}$ appears in $h_{1}$ or $h_{2}$. By Lemma $5.1,\left\langle\mathbf{x}_{\{1,2, \ldots, n\}}\right\rangle \subseteq\left\langle h_{1}\right\rangle \subseteq M$ or $\left\langle\mathbf{x}_{\{1,2, \ldots, n\}}\right\rangle \subseteq\left\langle h_{2}\right\rangle \subseteq N$. This shows that $M$ or $N$ should be equal to $L_{n}^{\mathbf{0}}(-m, n)$. Therefore, $L_{n}^{\mathbf{0}}(-m, j)$ is indecomposable.

\section{Simple modules $W_{n}^{\alpha}(m, J)$}

In this section, we investigate some submodules of the quotients

$$
L_{n}^{\boldsymbol{\alpha}}(m, j) / L_{n}^{\boldsymbol{\alpha}}(m, j-1) .
$$

We will assume $L_{n}^{\alpha}(m, j)=\{0\}$ for $j \leqslant-1$.

Definition 6.1. For $m \in \mathbb{Z}$ and a subset $J$ of $I_{\boldsymbol{\alpha}}$ with cardinality $j$, we define the following submodule of the quotient $L_{n}^{\boldsymbol{\alpha}}(m, j) / L_{n}^{\boldsymbol{\alpha}}(m, j-1)$

$$
W_{n}^{\boldsymbol{\alpha}}(m, J)=\left(V_{n}^{\boldsymbol{\alpha}}(m, J)+L_{n}^{\boldsymbol{\alpha}}(m, j-1)\right) / L_{n}^{\boldsymbol{\alpha}}(m, j-1) .
$$

We note that

$$
W_{n}^{\boldsymbol{\alpha}}(m, J) \cong V_{n}^{\boldsymbol{\alpha}}(m, J) /\left(V_{n}^{\boldsymbol{\alpha}}(m, J) \cap L_{n}^{\boldsymbol{\alpha}}(m, j-1)\right)
$$

and

$$
V_{n}^{\boldsymbol{\alpha}}(m, J) \cap L_{n}^{\boldsymbol{\alpha}}(m, j-1)=\sum_{J^{\prime}} V_{n}^{\boldsymbol{\alpha}}\left(m, J^{\prime}\right)
$$

where the summation runs over all $J^{\prime} \subset J$ with $\left|J^{\prime}\right|=j-1$. 
In $\S 5$, we saw that nontrivial modules

$$
L_{n}^{\boldsymbol{\alpha}}(m, 0) / L_{n}^{\boldsymbol{\alpha}}(m,-1) \cong L_{n}^{\boldsymbol{\alpha}}(m, 0)=V_{n}^{\boldsymbol{\alpha}}(m, \varnothing)
$$

are simple, and that for $m \geqslant 1$ the following quotient decomposes into simple submodules:

$$
L_{n}^{\mathbf{0}}(-m, 1) / L_{n}^{\mathbf{0}}(-m, 0) \cong \bigoplus_{\ell=1}^{n} V_{n}^{\mathbf{0}}(-m,\{\ell\}) .
$$

Let us generalize these observations.

Theorem 6.2. Let $m \in \mathbb{Z}$.

1) For $J \subset I_{\boldsymbol{\alpha}}$, the module $W_{n}^{\boldsymbol{\alpha}}(m, J)$ is simple.

2) For $1 \leqslant j \leqslant\left|I_{\boldsymbol{\alpha}}\right|$, the quotient module $L_{n}^{\boldsymbol{\alpha}}(m, j) / L_{n}^{\boldsymbol{\alpha}}(m, j-1)$ decomposes as

$$
L_{n}^{\boldsymbol{\alpha}}(m, j) / L_{n}^{\boldsymbol{\alpha}}(m, j-1)=\bigoplus_{J:|J|=j} W_{n}^{\boldsymbol{\alpha}}(m, J)
$$

where the direct sum is taken over all subsets $J$ of $I_{\boldsymbol{\alpha}}$ with cardinality $j$.

Proof. For Statement (1), for any nonzero element $\bar{f} \in W_{n}^{\boldsymbol{\alpha}}(m, J)$, we want to show that $\langle\bar{f}\rangle=W_{n}^{\boldsymbol{\alpha}}(m, J)$. From the definition of $W_{n}^{\boldsymbol{\alpha}}(m, J)$, we can assume that

$$
\bar{f}=f+L_{n}^{\boldsymbol{\alpha}}(m, j-1)
$$

where $f=\sum_{i=1}^{r} c_{i} \mathbf{x}^{\mathbf{k}_{i}} \in V_{n}^{\boldsymbol{\alpha}}(m, J)$ having distinct monomials $\mathbf{x}^{\mathbf{k}_{i}}$ in $V_{n}^{\boldsymbol{\alpha}}(m, J)$ with $\mathbf{k}_{i}^{n e g} \cap I_{\boldsymbol{\alpha}}=J$. From Lemma 5.1, $\langle f\rangle$ includes the cyclic modules $\left\langle\mathbf{x}^{\mathbf{k}_{i}}\right\rangle$. On the other hand, by Theorem 4.3, Theorem 4.4, Theorem 4.5, and Remark 4.6, each $\mathbf{x}^{\mathbf{k}_{i}}$ generates the module $V_{n}^{\boldsymbol{\alpha}}(m, J)$. This shows that $\langle\bar{f}\rangle=W_{n}^{\boldsymbol{\alpha}}(m, J)$.

For Statement (2), with (3.2) we see that

$$
\begin{gathered}
L_{n}^{\boldsymbol{\alpha}}(m, j) / L_{n}^{\boldsymbol{\alpha}}(m, j-1)=\left(\sum_{J:|J|=j} V_{n}^{\boldsymbol{\alpha}}(m, J)\right) / L_{n}^{\boldsymbol{\alpha}}(m, j-1) \\
=\left(\sum_{J:|J|=j} V_{n}^{\boldsymbol{\alpha}}(m, J)+L_{n}^{\boldsymbol{\alpha}}(m, j-1)\right) / L_{n}^{\boldsymbol{\alpha}}(m, j-1) .
\end{gathered}
$$

Therefore, we have

$$
L_{n}^{\boldsymbol{\alpha}}(m, j) / L_{n}^{\boldsymbol{\alpha}}(m, j-1)=\sum_{J:|J|=j} W_{n}^{\boldsymbol{\alpha}}(m, J)
$$


where the summation is over $J \subset I_{\boldsymbol{\alpha}}$ with $|J|=j$. Now, suppose we have

$$
\bar{f} \in W_{n}^{\boldsymbol{\alpha}}\left(m, J_{1}\right) \cap W_{n}^{\boldsymbol{\alpha}}\left(m, J_{2}\right)
$$

with distinct subsets $J_{1}$ and $J_{2}$ of $I_{\boldsymbol{\alpha}}$. Then, we can assume that $\bar{f}=$ $f+L_{n}^{\alpha}(m, j-1)$ where $f=\sum_{i=1}^{r} c_{i} \mathbf{x}^{\mathbf{k}_{i}}$ with distinct monomials $\mathbf{x}^{\mathbf{k}_{i}}$ such that $\mathbf{k}_{i}^{\text {neg }} \cap I_{\boldsymbol{\alpha}} \subseteq J_{1} \cap J_{2}$ for all $i$. Since $\left|J_{1} \cap J_{2}\right|<j$, this shows that $f \in$ $L_{n}^{\boldsymbol{\alpha}}(m, j-1)$ and therefore $\bar{f}$ is zero in the quotient $L_{n}^{\boldsymbol{\alpha}}(m, j) / L_{n}^{\boldsymbol{\alpha}}(m, j-1)$. Therefore, we obtain the direct sum expression in the statement.

Next we investigate the cases when $W_{n}^{\alpha}(m, J)$ are highest weight modules.

Theorem 6.3 (Highest weight vector in $W_{n}^{\boldsymbol{\alpha}}(m, J)$ ).

1) For an integer $1 \leqslant \ell \leqslant n$, if $\boldsymbol{\alpha} \in \mathbb{C}^{n}$ is such that $\boldsymbol{\alpha}[\ell]=c$ is nonzero and $\boldsymbol{\alpha}\left[\ell^{\prime}\right]=0$ for all $\ell^{\prime} \neq \ell$ and $J=\{1,2, \ldots, \ell-1\} \subseteq I_{\boldsymbol{\alpha}}$, then for every $m \in \mathbb{Z}$ the module $W_{n}^{\boldsymbol{\alpha}}(m, J)$ is a highest weight module having a highest weight vector

$$
\left(x_{1}^{-1} x_{2}^{-1} \cdots x_{\ell-1}^{-1} x_{\ell}^{m+\ell-1}\right)+L_{n}^{\alpha}(m, \ell-2)
$$

with highest weight

$$
(-1,-1, \ldots,-1, m+\ell-1+c, 0, \ldots, 0) .
$$

2) Let $\boldsymbol{\alpha}=\mathbf{0}$ and therefore $I_{\boldsymbol{\alpha}}=\{1,2, \ldots, n\}$. For $1 \leqslant \ell \leqslant n$, if $J=\{1,2, \ldots, \ell-1\}$ then for $m \in \mathbb{Z}$ such that $m+\ell-1 \geqslant 0$ the module $W_{n}^{\mathbf{0}}(m, J)$ is a highest weight module having a highest weight vector

$$
\left(x_{1}^{-1} x_{2}^{-1} \cdots x_{\ell-1}^{-1} x_{\ell}^{m+\ell-1}\right)+L_{n}^{\mathbf{0}}(m, \ell-2)
$$

with highest weight

$$
(-1,-1, \ldots,-1, m+\ell-1,0, \ldots, 0) .
$$

In particular, if $\ell=1$ and $J=\varnothing$ then for $m \geqslant 0$, the module $W_{n}^{\mathbf{0}}(m, \varnothing)$ is a $(m+1)$-dimensional module with highest weight $(m, 0, \ldots, 0)$.

3) Let $\boldsymbol{\alpha}=\mathbf{0}$ and therefore $I_{\boldsymbol{\alpha}}=\{1,2, \ldots, n\}$. For $1 \leqslant \ell \leqslant n$, if $J=\{1,2, \cdots, \ell\}$ then for $m \in \mathbb{Z}$ such that $m+\ell-1<0$ the module $W_{n}^{\mathbf{0}}(m, J)$ is a highest weight module having a highest weight vector

$$
\left(x_{1}^{-1} x_{2}^{-1} \cdots x_{\ell-1}^{-1} x_{\ell}^{m+\ell-1}\right)+L_{n}^{\mathbf{0}}(m, \ell-1)
$$


with highest weight

$$
(-1,-1, \ldots,-1, m+\ell-1,0, \ldots, 0) .
$$

In particular, if $\ell=n$ and $J=\{1,2, \ldots, n\}$, then for $m \leqslant-n$ the module $W_{n}^{\mathbf{0}}(m,\{1,2, \ldots, n\})$ is a finite dimensional module with highest weight

$$
(-1,-1, \ldots,-1, m+n-1) .
$$

Proof. We first notice that the given elements $\mathbf{x}^{\mathbf{k}}+L_{n}^{\boldsymbol{\alpha}}(m, j-1)$ generate $W_{n}^{\boldsymbol{\alpha}}(m, J)$ where $j=|J|$ (see Theorem 4.3, Theorem 4.4, and Theorem 4.5). It is straightforward to verify their weights under the action of the Cartan subalgebra of $\mathfrak{g l}(n)$ generated by $E_{a a}$ for $1 \leqslant a \leqslant n$. Therefore, now it is enough to show that

$$
\begin{aligned}
E_{a b} \cdot\left(\mathbf{x}^{\mathbf{k}}+L_{n}^{\boldsymbol{\alpha}}(m, j-1)\right) & =(\mathbf{k}[b]+\boldsymbol{\alpha}[b])\left(x_{a} x_{b}^{-1}\right) \mathbf{x}^{\mathbf{k}}+L_{n}^{\boldsymbol{\alpha}}(m, j-1) \\
& =L_{n}^{\boldsymbol{\alpha}}(m, j-1)
\end{aligned}
$$

in $W_{n}^{\boldsymbol{\alpha}}(m, J)$ for all $1 \leqslant a<b \leqslant n$.

For Statement (1), if $a<b$ and $b \geqslant \ell+1$, then since $\mathbf{k}[b]=\boldsymbol{\alpha}[b]=0$ we have

$$
E_{a b} \cdot\left(x_{1}^{-1} \cdots x_{\ell-1}^{-1} x_{\ell}^{m+\ell-1}\right)=(0+0)\left(x_{a}^{1} x_{b}^{-1}\right)\left(x_{1}^{-1} \cdots x_{\ell-1}^{-1} x_{\ell}^{m+\ell-1}\right)=0 .
$$

If $a<b$ and $b \leqslant \ell$, then $a \leqslant \ell-1$ and

$$
E_{a b} \cdot\left(x_{1}^{-1} \cdots x_{\ell-1}^{-1} x_{\ell}^{m+\ell-1}\right)=(\mathbf{k}[b]+\boldsymbol{\alpha}[b])\left(x_{a}^{1} x_{b}^{-1}\right)\left(x_{1}^{-1} \cdots x_{\ell-1}^{-1} x_{\ell}^{m+\ell-1}\right)
$$

where $\mathbf{k}[b]=-1$ and $\boldsymbol{\alpha}[b]=0$ if $b \leqslant \ell-1$; and $\mathbf{k}[b]=m+\ell-1$ and $\boldsymbol{\alpha}[b]=c$ if $b=\ell$. Writing $\mathbf{x}^{\mathbf{q}}$ for the monomial in the right hand side, we see that $\mathbf{q}[a]=0$ because $a \leqslant \ell-1$ and therefore $\left|\mathbf{q}^{n e g} \cap I_{\boldsymbol{\alpha}}\right|<\ell-1$. This shows that $\mathbf{x}^{\mathbf{q}} \in L_{n}^{\alpha}(m, \ell-2)$ and therefore (6.1) is true.

For Statement (2), the first part can be shown similarly to the previous case. The second part with the conditions $\ell=1$ and $J=\varnothing$ follows directly from Definition 6.1 with $L_{n}^{\mathbf{0}}(m,-1)=\{0\}$ and Theorem 5.4 (2).

For Statement (3), if $a<b$ and $b \geqslant \ell+1$, then since $\mathbf{k}[b]=\boldsymbol{\alpha}[b]=0$ we have

$$
E_{a b} \cdot\left(x_{1}^{-1} \cdots x_{\ell-1}^{-1} x_{\ell}^{m+\ell-1}\right)=(0+0)\left(x_{a}^{1} x_{b}^{-1}\right)\left(x_{1}^{-1} \cdots x_{\ell-1}^{-1} x_{\ell}^{m+\ell-1} x_{b}^{-1}\right)=0 .
$$

If $a<b$ and $b \leqslant \ell$, then since $\boldsymbol{\alpha}[b]=0$ we have

$$
E_{a b} \cdot\left(x_{1}^{-1} \cdots x_{\ell-1}^{-1} x_{\ell}^{m+\ell-1}\right)=\mathbf{k}[b]\left(x_{a}^{1} x_{b}^{-1}\right)\left(x_{1}^{-1} \cdots x_{\ell-1}^{-1} x_{\ell}^{m+\ell-1}\right)
$$


where $\mathbf{k}[b]=-1$ if $b \leqslant \ell-1$ and $\mathbf{k}[b]=m+\ell-1$ if $b=\ell$. Again, by denoting the monomial in the right hand side by $\mathbf{x}^{\mathbf{q}}$, we see that $\mathbf{q}[a]=0$ because $a \leqslant \ell-1$, and therefore $\left|\mathbf{q}^{\text {neg }} \cap I_{\boldsymbol{\alpha}}\right|<\ell$. This shows that $\mathbf{x}^{\mathbf{q}} \in L_{n}^{\boldsymbol{\alpha}}(m, \ell-1)$ and therefore (6.1) is true.

We note that the highest weights of $W_{n}^{\boldsymbol{\alpha}}(m, J)$ given in Theorem 6.3 are integral dominant (see, for example, [5, §3]) only when

i) $\boldsymbol{\alpha}=\mathbf{0}, J=\varnothing$, and $m \geqslant 0$;

ii) $\boldsymbol{\alpha}=\mathbf{0}, J=\{1,2, \ldots, n\}$, and $m \leqslant-n$.

Indeed, one can easily check that these are the only cases when the modules $W_{n}^{\boldsymbol{\alpha}}(m, J)$ are finite dimensional.

\section{References}

[1] D. J. Britten and F. W. Lemire, A classification of simple Lie modules having a 1-dimensional weight space. Trans. Amer. Math. Soc. 299 (1987), no. 2, 683-697.

[2] K. A. Brown and K. R. Goodearl, Lectures on algebraic quantum groups. Advanced Courses in Mathematics. CRM Barcelona. Birkhäuser Verlag, Basel, 2002.

[3] S. Eswara Rao, Representations of Witt algebras. Publ. Res. Inst. Math. Sci. 30 (1994), no. 2, 191-201.

[4] S. Eswara Rao, Irreducible representations of the Lie-algebra of the diffeomorphisms of a d-dimensional torus. J. Algebra 182 (1996), no. 2, 401-421.

[5] R. Goodman and N. R. Wallach, Symmetry, representations, and invariants. Graduate Texts in Mathematics, 255. Springer, Dordrecht, 2009.

[6] X. Guo and K. Zhao, Irreducible weight modules over Witt algebras. Proc. Amer. Math. Soc. 139 (2011), no. 7, 2367-2373.

\section{Cheonho Choi,} Sangjib Kim

\section{Haeyun Seo}

\section{CONTACT INFORMATION}

\author{
Department of Mathematics, Korea University, \\ 145 Anam-ro Seongbuk-gu, Seoul 02841, South \\ Korea
E-Mail(s): cheonho@korea.ac.kr, sk23@korea.ac.kr

Department of Mathematics, University of Maryland, William E. Kirwan Hall, 4176

Campus Drive, College Park, MD 20742-4015, USA

E-Mail(s): hys2500@umd.edu

Received by the editors: 13.12.2018

and in final form 24.02.2021. 